\title{
EDITORIAL
}

\section{What do we learn from HER2-positive breast cancer genomic profiles?}

\author{
Charles Theillet* \\ See related research by Staaf et al., http://breast-cancer-research.com/content/12/3/R25
}

\begin{abstract}
Patients with a tumor presenting amplification of the HER2 gene are currently offered trastuzumab (herceptin) and this has greatly changed their outcome. However, a number of HER2-positive cancers show intrinsic or acquired resistance to trastuzumab and there are clear indications that they form a heterogeneous group of tumors. A paper in this issue of Breast Cancer Research addresses this heterogeneity at the genomic level.
\end{abstract}

HER2 is the only molecular marker in breast cancer to make its way into clinical practice. This protein kinase receptor has become a therapeutical target since the development by Genentech (South San Francisco, CA, USA) of anti-HER2 monoclonal antibodies. But do HER2-positive breast cancers form a homogeneous group of tumors? This question is addressed by Staaf and colleagues in this issue of Breast Cancer Research [1].

Amplification and overexpression of the HER2 gene in about $15 \%$ of breast cancers were originally discovered in 1987 [2,3], in the early days of molecular oncology. Since that time, technologies have changed, the human genome has been sequenced, and DNA arrays allow investigators to address genetic changes on a genome-wide scale. Our views of breast cancer and of the sequence of oncogenic changes that govern its emergence have changed considerably as well.

For HER2-positive breast cancers, the introduction of trastuzumab has been a radical change, improving the outcome of these patients substantially. This success has contributed to the definition of a HER2-positive breast cancer subclass. However, a number of patients show either primary or acquired resistance to this therapy. This clinical reality motivated the group of Ake Borg [1] at the

*Correspondence: charles.theillet@inserm.fr

IRCM, INSERM U896, CRLC Val d'Aurelle, F34298 Montpellier, France
University of Lund, Sweden, to address the question of the biological homogeneity of HER2-positive tumors. To do this, they collaborated with colleagues from the University of Iceland in the collection of a large (200) series of primary HER2-positive breast cancers, which they characterized by CGH (comparative genomic hybridization) using tiling-path BAC (bacterial artificial chromosome) array. These data were related to an external dataset comprising CNA (copy number alteration) profiles from 554 HER2-negative breast tumors. The principal finding of this work is that HER2-positive breast cancers present a large diversity of anomalies affecting most of the genome. They show, apart from HER2 amplification, high-level amplification at multiple sites and a number of these amplifications correspond to known oncogenes (FGFR1, MYC, CCND1, and ZNF217). Some apparently distinguishing features of HER2-positive breast tumors are the so-called firestorm patterns. Firestorms correspond to multiple focal amplifications of neighboring loci. These occurred on a number of chromosomal arms but principally at $17 \mathrm{q}$. Such patterns concentrated on the chromosomal arm carrying the HER2 locus raise interesting questions about the mechanism at work. The first idea that comes to mind is that it may correspond to a cooperative mechanism resulting in the selection of secondary mutations. As is often the case, the reality is more complex since only a few of these firestorm amplicons at 17q were recurrent. The second important finding concerns amplification of the HER2 locus, which can be split into two subsets: (a) narrow amplicons (86 kb in its smallest form) that centered around HER 2 and that comprise 6 to 10 genes, of which 4 showed recurrent overexpression, and (b) a large amplicon $(1.1 \mathrm{Mb})$ starting centromeric to $H E R 2$ and extending telomeric to the TOP $2 A$ gene. The abrupt break downstream of TOP $2 A$ is a clear indication of a coselection. This work shows that these two amplification patterns point to different breast cancer subsets. The small amplicon is found in estrogen receptor (ER)negative tumors, whereas $H E R 2 / T O P 2 A$ coamplification is correlated with ER-positive tumors and a better survival. There are other signs that HER2-positive tumors 
divide according to the ER status. HER2 ${ }^{+} / \mathrm{ER}^{-}$and HER2 ${ }^{+} /$ $\mathrm{ER}^{+}$presented different patterns of anomalies; in particular, loss at $5 \mathrm{q}$ was limited to $\mathrm{ER}^{-}$and amplification at $11 \mathrm{q} 13$ was limited to $\mathrm{ER}^{+}$. HER2 ${ }^{+} / \mathrm{ER}^{-}$showed higher ploidy indices and profiles that were more rearranged than those of their $\mathrm{ER}^{+}$counterparts.

This work parallels and complements an earlier study by the same group [4]; that study was based on expression profiling and aimed at determining a molecular predictor of bad outcome in HER2-positive tumors. A total of 58 HER2-positive breast cancers were analyzed. By unsupervised analysis, three clusters of tumors, one of which presented a substantially worse outcome, were delineated. A 158-gene prognostic predictor specific to HER2positive breast cancer was proposed.

Altogether, these data bring clear evidence that HER2positive breast tumors do not form a homogeneous biological entity. This was already apparent in molecular subtypes defined by unsupervised expression profiling classification. Four to five molecular subtypes, one of which was the ERBB2 subtype, were described [5]. It was soon apparent that this ERBB2 subtype was not exclusively composed of HER2-amplified breast tumors and, conversely, that HER2-positive tumors clustered in other molecular subgroups. This was very clearly confirmed by Weigelt and colleagues [6] in a recent study (2010) comparing the relative efficiency of two singlesample predictors (SSPs). There are repeated calls for a refined molecular classification, but there is a reasonable likelihood that it would not delineate a subgroup corresponding to HER2-positive cancers.

If HER2-positive tumors do not correspond to a biological subclass of breast cancer, they define a clinical subset of patients. This determines its importance and the huge efforts that are deployed to better characterize them. It must be mentioned that in the framework of the International Cancer Genome Consortium (ICGC), which aims at sequencing the genome of 500 tumors of at least 50 cancer types, France has decided to take HER2positive breast cancer as a target. The genomic heterogeneity revealed by the work of Staaf and colleagues [1] calls for further work in order to determine the lines along which we will delineate the different subsets of HER2-positive breast cancers. It will be interesting, in particular, to see whether it is possible to outline a coherent scheme of co-occurring amplifications. If such a scheme exists, it may be a good basis on which to define complementary therapies by targeting genes coamplified with HER2. There are great expectations that the ICGC program will bring answers to these questions.

Abbreviations

ER, estrogen receptor; ICGC, International Cancer Genome Consortium.

Competing interests

The author declares that he has no competing interests.

Published: 1 June 2010

References

1. Staaf J, Jönsson G, Ringnér M, Vallon-Christersson J, Grabau D, Arason A, Gunnarsson H, Agnarsson BA, Malmström P-O, Johannsson OT, Loman N, Barkardottir RB, Borg A: High-resolution genomic and expression analyses of copy number alterations in HER2-amplified breast cancer. Breast Cancer Res 2010, 12:R25.

2. Kraus MH, Popescu NC, Amsbaugh SC, King CR: Overexpression of the EGF receptor-related proto-oncogene erbB-2 in human mammary tumor cell lines by different molecular mechanisms. Embo J 1987, 6:605-610.

3. Slamon DJ, Clark GM, Wong SG, Levin WJ, Ullrich A, McGuire WL: Human breast cancer: correlation of relapse and survival with amplification of the HER-2/neu oncogene. Science 1987, 235:177-182.

4. Staaf J, Ringnér M, Vallon-Christersson J, Jönsson G, Bendahl PO, Holm K, Arason A, Gunnarsson H, Hegardt C, Agnarsson BA, Luts L, Grabau D, Fernö M, Malmström PO, Johannsson OT, Loman N, Barkardottir RB, Borg A: Identification of subtypes in human epidermal growth factor receptor 2-positive breast cancer reveals a gene signature prognostic of outcome. J Clin Oncol 2010, 28:1813-1820.

5. Sorlie T, Tibshirani R, Parker J, Hastie T, Marron JS, Nobel A, Deng S, Johnsen H, Pesich R, Geisler S, Demeter J, Perou CM, Lønning PE, Brown PO, BørresenDale AL, Botstein D: Repeated observation of breast tumor subtypes in independent gene expression data sets. Proc Natl Acad Sci U S A 2003, 100:8418-8423.

6. Weigelt B, Mackay A, A'Hern R, Natrajan R, Tan DSP, Dowsett M, Ashworth A, Reis-Filho JS: Breast cancer molecular profiling with single sample predictors: a retrospective analysis. Lancet Oncol 2010, 11:339-349.

doi:10.1186/bcr2571

Cite this article as: Theillet $\mathrm{C}$ : What do we learn from HER2-positive breast cancer genomic profiles? Breast Cancer Research 2010, 12:107. 